LAWRENCE LIVERMORE N A T IO N A L LABORATORY

\section{Are collapsars responsible for some r-process elements? How could we tell?}

J. Pruet

April 6, 2004

1st Joint MSU/Argonne/INT RIA Workshop Seattle, WA, United States January 7, 2004 through January 11, 2004 
This document was prepared as an account of work sponsored by an agency of the United States Government. Neither the United States Government nor the University of California nor any of their employees, makes any warranty, express or implied, or assumes any legal liability or responsibility for the accuracy, completeness, or usefulness of any information, apparatus, product, or process disclosed, or represents that its use would not infringe privately owned rights. Reference herein to any specific commercial product, process, or service by trade name, trademark, manufacturer, or otherwise, does not necessarily constitute or imply its endorsement, recommendation, or favoring by the United States Government or the University of California. The views and opinions of authors expressed herein do not necessarily state or reflect those of the United States Government or the University of California, and shall not be used for advertising or product endorsement purposes. 


\title{
ARE COLLAPSARS RESPONSIBLE FOR SOME R-PROCESS ELEMENTS? HOW COULD WE TELL?
}

\author{
J. PRUET \\ $N$-Division Lawrence Livermore National Lab \\ 7000 E. Ave, \\ Livermore, CA 94550, USA \\ E-mail: pruet1@llnl.gov
}

\begin{abstract}
We consider the possibility that supernovae which form hyper-accreting black holes might be responsible for synthesis of $\mathrm{r}$-process elements with mass $\mathrm{A} \lesssim 130$. Calculations are presented which show that these elements are naturally synthesized in neutron-rich magnetically-dominated bubbles born in the inner regions of a black hole accretion disk. Simple considerations suggest that the total mass ejected in the form of these bubbles is about that needed to account for the entire galactic inventory of the 2nd-peak r-process elements. We also argue that if collapsars are responsible for, e.g., Ag synthesis, then $\mathrm{Ag}$ abundances should be correlated with Sc and/or Zn abundances in metal-poor stars.
\end{abstract}

\section{Introduction}

There is growing evidence that the engines powering long-duration Gamma Ray Bursts (GRBs) are associated with interesting nucleosynthesis. Observations of a characteristic 'red bump' in late-time GRB light curves indicate that many, maybe all, long GRBs copiously produce radioactive nickel moving outwards at fractions of the speed of light ${ }^{1,2,3,4}$. This ${ }^{56} \mathrm{Ni}$ is familiar as the product of explosive burning occurring as a strong shock sweeps through the stellar mantle in ordinary SNe. In the still mysterious GRB environment, however, the $\mathrm{Ni}$ is likely telling us about a unique and different process. Consideration of this will lead to interesting insights about a possible origin for the r-process elements.

Though there are other possibilities, we will assume that GRBs are produced by a viscous black hole accretion disk formed after the collapse of a rotating massive $\operatorname{star}^{5,6}$. Within the context of this collapsar model there are two ways observed nickel could be synthesized. As in 'successful' SNe, Ni may be synthesized explosively as a strong shock traverses the stellar mantle. One difficulty with this idea is that there is no clear analog of the 
neutron star core bounce that initiates strong shocks in typical SNe. Also, even if some mechanism does initiate a strong shock, the parameters of this mechanism need to be very finely chosen in order to explain observations ${ }^{7}$.

Observed nickel might also be synthesized in a vigorous wind blown off the accretion disk surface ${ }^{6,8}$. Here we focus on this possibility. In the next two sections we discuss the composition of the accretion disk and the nucleosynthesis occurring in winds blown from the disk. It turns out that though the disk itself is likely quite neutron rich, it is hard to see how this neutron richness can survive in the wind. So, r-process elements cannot be synthesized in a wind. Section 3 describes a different way of synthesizing heavy, neutron-rich elements - localized bubbles. Section 4 outlines an observational test of whether or not GRBs are responsible for synthesizing some r-process elements.

\section{Composition of the accretion disk}

Before we can understand nucleosynthesis in collapsars, we need to know conditions in the disk material feeding outflows. At large radii the disk is fed by relatively cool bound nuclei found in the stellar mantle. This material has an electron fraction very close to $1 / 2$. Here the electron fraction $Y_{e}=$ $p /(n+p)$, with $p$ and $n$ the number densities of protons and neutrons. As material spirals inwards, viscous heating converts a fraction of the liberated gravitational energy into thermal energy. At a radius of about $10^{8} \mathrm{~cm}$ the disk becomes hot enough that $\alpha$-particles and all heavier elements dissociate into free nucleons. Once this happens the charged lepton capture reactions

$$
\begin{aligned}
& e^{-} \mathrm{p} \rightarrow \mathrm{n} \nu_{e} \\
& e^{+} \mathrm{n} \rightarrow \mathrm{p} \bar{\nu}_{e}
\end{aligned}
$$

begin in earnest. The neutron to proton ratio is then set by a competition between the above processes.

The composition of the inner disk is a sensitive function of the rate $\dot{M}$ at which the disk is accreting onto the black hole and the strength of the viscosity, described here by the usual parameter $\alpha$. Figure 1 shows the composition of a disk characterized by $\dot{M}=0.03 M_{\odot} /$ sec and $\alpha=0.1$ accreting onto a hole of mass $3 M_{\odot}$. At small radii neutrino losses become important and sap entropy from the flow. This inner region is characterized by a large electron degeneracy and low electron fraction $\left(Y_{e} \approx 0.15\right)$.

Figure 1 was taken from Pruet, Woosley \& Hoffman 2003. That work used published disk structures ${ }^{10}$ to calculate the disk composition. A somewhat different treatment of the disk composition appeared at about the 


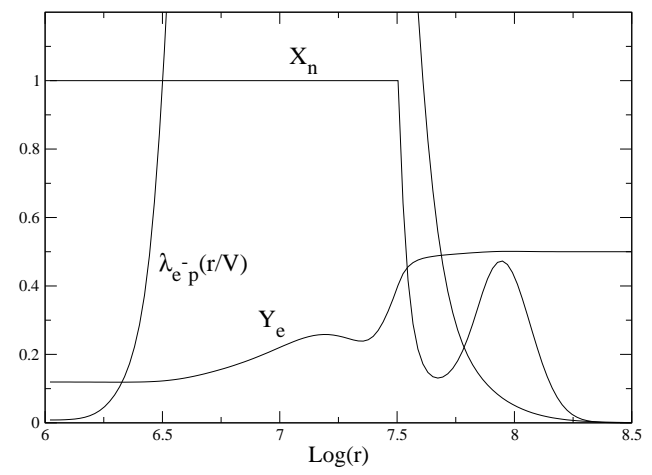

Figure 1. Composition of an accretion disk characterized by $\dot{M}=0.1 M_{\odot} /$ sec and $\alpha=0.03$. Here $X_{\mathrm{n}}$ is the mass fraction of free nucleons and $\lambda_{e^{-} \mathrm{p}}(r / V)$ is a rough instantaneous estimate of the number of $e^{ \pm}$captures suffered by a nucleon before the nucleon crosses the event horizon. Units for $r$ here are $\mathrm{cm}$.

same time by Beloborodov ${ }^{11}$. Beloborodov develops a useful, semi-analytic, and in some sense self-consistent description of the disk structure and composition.

There is basic agreement among different authors that the inner regions of the disk become markedly neutron rich for modest accretion rates $\left(\dot{M} \gtrsim 0.1 \mathrm{M}_{\odot} / \mathrm{sec}\right)$. However, there remain a number of shortcomings in the understanding of accretion disk composition. In the first place, previous efforts are based on 1-d height-integrated approximations for the disk structure. The dependence of $Y_{e}$ on height within the disk, or an adequate understanding of how $Y_{e}$ tends towards the disk surface, has yet to be worked out (though preliminary studies have been made ${ }^{12,8}$ ). Secondly, if disk winds really are responsible for observed nickel, then about half of the accreting material must be blown away before it has a chance to get to the black hole. The influence of this mass loss on the disk structure has been studied for a few cases $^{6}$. Feedback on the disk composition remains more or less unstudied.

\section{Winds from the Disk}

MacFadyen \& Woosley first calculated that viscous heating above the surface of a disk could deposit entropy and drive a wind. Their observation that nickel might be synthesized in this wind presaged observations of SN-like light curves associated with ordinary GRBs. Calculations and observations of GRBs imply that the wind is very energetic. For SN2003dh, for example, 
a total outflow kinetic energy of a few times $10^{52} \mathrm{erg}$ was inferred ${ }^{1,13}$. This is an order of magnitude larger than the kinetic energy associated with typical SNe and dwarves the energy in the GRB proper. The large energy carried by the wind implies that the overlying stellar mantle is quickly swept away and can be neglected at the small radii where nuclei are synthesized. This is an important observation because it allows simple treatments of the wind near the central regions of the star.

To motivate a discussion of the wind properties we give here a very schematic prescription for calculating the wind:

$$
\begin{aligned}
T d s & =d Q=(\nu \text { heating }+ \text { viscous heating }- \text { neutrino losses }) \\
d Y_{e} & =\left(\text { effect of } \mathrm{e}^{ \pm} / \nu \text { capture }\right) \\
d v & =(\text { pressure gradient }+ \text { "centrifugal" forces }+ \text { gravity })
\end{aligned}
$$

Here $s$ is the entropy per baryon and $v$ is the velocity projected along fluid streamlines. Let's compare these terms with their counterparts describing spherically symmetric winds from neutron stars.

i) The entropy equation: One interesting result from Qian and Woosley's ${ }^{14}$ study of outflows from neutron stars (NSs) was that the wind properties important for nucleosynthesis are quite insensitive to the heating rate (i.e neutrino energy deposition rate). For example, those authors found that the asymptotic entropy should scale as $L_{\nu}^{-1 / 6}$, with $L_{\nu}$ the $\nu_{e}$ or $\bar{\nu}_{e}$ luminosity. In the case of winds from NSs, this insensitivity arises in part because there is an important interplay between the neutrino luminosity and conditions at the base of the wind. At the base of the wind the temperature adjusts itself so that the neutrino heating and neutrino cooling rates balance each other.

Things are more complicated for outflows from accretion disks. If a simple $\alpha$-prescription for the viscosity is used it is found that viscous heating may or may not dominate over neutrino heating, depending on fine details such as the angular momentum of the black hole. Also, there is in general not a tight balance between heating and cooling in the disk. Nonetheless, it has been found that the asymptotic wind entropy is not sensitive to factors of $\sim 3$ changes in the heating rates ${ }^{8}$. This is comforting because it is generally agreed that "viscous heating" - represented by a shear-stress tensor - is only a very rough representation of the magnetic processes providing viscosity. Because wind properties are not so sensitive to the heating rates, it may be that one can entirely neglect either the neutrino heating or the viscous heating (though not both at the same time) in a description of the wind and get close to the right answer for the asymptotic wind properties. 
ii) The $Y_{e}$ equation: In winds from neutron stars the electron fraction is set by a competition between $\nu_{e}$ and $\bar{\nu}_{e}$ capture. Apart from effects having to do with nucleosynthesis, the asymptotic $Y_{e}$ just mirrors the neutrino spectrum coming from the NS. Because the neutron star through which the neutrinos diffuse is neutron-rich, the $\nu_{e}$ spectrum is cooler than the $\bar{\nu}_{e}$ spectrum. Consequently, $\overline{\nu_{e}} \mathrm{p} \rightarrow \mathrm{n}$ wins out over $\nu_{e} \mathrm{n} \rightarrow \mathrm{p}$, and the outflow is driven neutron-rich.

In winds from accretion disks all factors conspire to drive the outflow proton rich. In part this is because $e^{ \pm}$capture generally dominates over neutrino capture. Once the entropy of the outflow is raised by viscous heating, the electron degeneracy is removed and positron capture wins out over electron capture because of the neutron-proton mass difference. When neutrinos are important they also tend to drive the outflow proton rich because collapsar-like disks are generally not optically thick to neutrinos and the most energetic neutrinos come from $e^{-} \mathrm{p} \rightarrow \mathrm{n} \bar{\nu}_{e}$.

iii) Evolution of velocity along fluid streamlines. This is straightforward in the case of spherically symmetric winds from NSs. For winds from disks, this equation seems to kill the possibility of a simple 1-d treatment of the wind. To see why, note that within the disk the azimuthal velocity is essentially Keplerian, so the material already has half the kinetic energy needed to escape the gravitational pull of the black hole. Now, what is the evolution of azimuthal velocity with height above the disk? The answer to this question depends sensitively on the $\theta-\phi$ component of the shear stress tensor, which in turn depends on details of the magnetic processes responsible for viscosity. There are two limiting assumptions. One is that fluid streamlines are frozen into and co-rotate with the disk. In this case no heating is needed to drive material from the disk and the asymptotic entropy can be very low. The other limiting assumption is that the azimuthal velocity reaches the asymptotic value of zero within a few disk-scale heights of the disk mid-plane. In this case the asymptotic entropies are found to be $\sim 30-50$. It is clear from a consideration of the magnetic field strengths needed to anchor streamlines to the disk that the second limiting approximation is closer to the truth.

To summarize results of previous studies of disk-winds in broad stroke, asymptotic entropies are modest $(\lesssim 30-50)$ and the asymptotic $Y_{e}$ is never lower than about 0.45 . There is actually a strong anti-correlation between $Y_{e}$ in the disk and $Y_{e}$ at infinity. Heavy neutron rich elements can't be synthesized in winds. 


\section{Bubbles}

If accretion disks really were characterized by some sort of friction-like microscopic viscosity, then only proton-rich modest entropy outflows would occur. However, it is generally agreed that a magnetic instability, the so called magneto-rotational instability ${ }^{15}$, is responsible for mediating angular momentum transfer in the disk. Because of this, one expects there to be small, localized regions of the disk which have larger than average magnetic field densities. Pressure equilibrium with the ambient fluid implies that these bubbles will be underdense and will rise in the approximately exponential atmosphere of the disk. The time it takes to rise one disk scaleheight is roughly a Kepler period, or about a few ms. This is much faster than the rise time of the wind. The electron capture timescale in the disk is

$$
\tau_{\text {ec }} \approx 0.1 \sec (3 \mathrm{MeV} / T)^{5},
$$

which implies that magnetically dominated bubbles retain their neutronrichness. As a bubble rises magnetic reconnection can convert energy in magnetic fields to thermal energy and in this way increases the entropy of the bubble.

In the dynamic collapsar environment there will likely be a wide variety of these bubbles. Without detailed simulations only a few things are known. The electron fraction is somewhere between that characterizing the disk and that characterizing the wind. The asymptotic entropy of the bubbles must be at least as large as that characterizing the wind, otherwise bubbles wouldn't rise, but would instead break early on and mix with the wind. Lastly, the dynamic time scale characterizing the evolution of the wind is likely not much different from the dynamic time scale characterizing the evolution of the bubbles, because both the wind and the bubbles are radiation dominated. For a radiation dominated fluid the pressure is approximately independent of the density. To incorporate these general considerations, we can describe bubbles by

$$
s=50+50 r ; Y_{e}=0.15+0.25 r ; \tau=0.03(1+4 r) \mathrm{sec} ; T_{9, \text { mix }}=1+2 r .
$$

Here $r$ is a random number chosen separately for each of the above expressions and $T_{9, \text { mix }}$ is the temperature at which the bubble is assumed to break and mix with the wind material.

Average overproduction factors for one hundred bubbles generated as described above are shown in Fig. 2. Overall there is quite good agreement with the solar abundance pattern of $90<A<130 r$-process elements. 


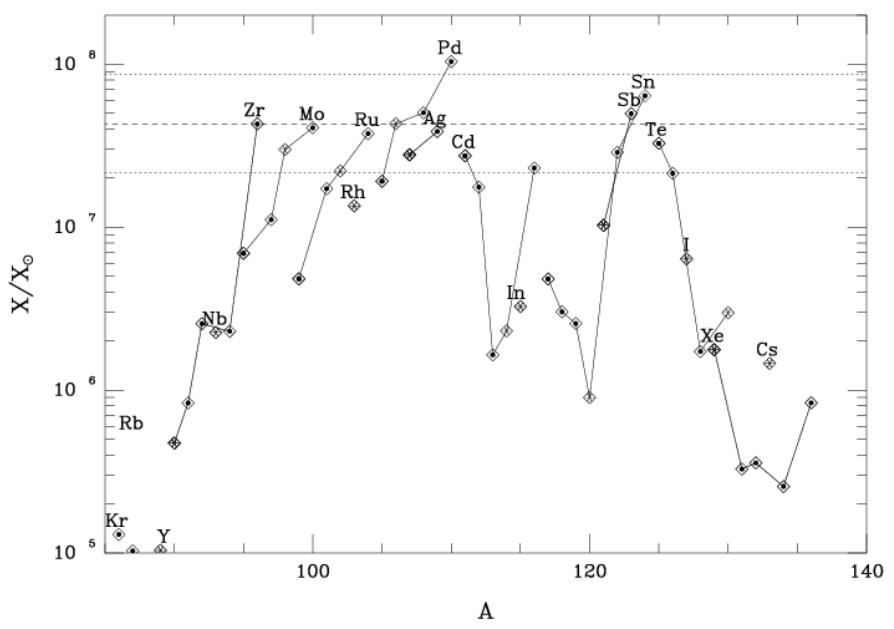

Figure 2. Average overproduction factors for 100 bubbles generated according to eqn. (7). Solid lines connect isotopes of a given element. The most abundant isotope in the Sun for a given element is plotted as an asterisk. A diamond around a data point indicates the production of that isotope as a radioactive progenitor. Agreement with the solar abundance pattern of $r$-process elements with $A<130$ is quite good, though ${ }^{127} \mathrm{I}$ is under-produced by a factor of about 4 . Production of species heavier than $A=130$ is negligible.

If collapsars are responsible for the 2nd peak r-process elements then they must eject $M_{b} \approx 10^{-5} M_{\odot} / f_{c}$ in the form of bubbles in order to account for the present day inventory of these elements in the galaxy. Here $f_{c}$ is the fraction of core collapse SNe that become collapsars. The collapsar rate is unknown, but is likely $\sim 0.1-0.01$ as large as the SNII rate ${ }^{16}$. The total mass needed in the form of bubbles is less than about $0.1 \%$ the total wind mass. If typical bubbles are formed with initial radius $r_{b}$, temperature $T_{b}$, and entropy $s_{b}$ per baryon, the amount of mass needed in the form of bubbles implies that the number of bubbles needed per event is

$$
n_{b} \approx 500 \frac{s_{b}}{50}\left(\frac{10^{6} \mathrm{~cm}}{r_{b}}\right)^{3}\left(\frac{2 \mathrm{MeV}}{T_{b}}\right)^{3} \frac{M_{b}}{10^{-4} M_{\odot}} .
$$

Now, if the disk lasts for a time $t$ comparable to observed durations of GRBs, the number of disk revolutions, or magnetic field windings, per bubble is

$$
n_{\text {wind }}=\frac{t \Omega_{k}}{2 \pi n_{b}} \approx 8 \frac{t}{50 \sec } \frac{\Omega_{k}}{10^{3} \sec ^{-1}} \frac{10^{3}}{n_{b}}
$$

which is a reasonable number if magnetic instabilities take a few rotations 
to develop.

\section{How could we tell?}

There don't seem to be any strong arguments against collapsars as the site of $A \lesssim 130$ r-process elements. In fact, Qian and his collaborators have argued for some time that the 2nd-peak elements are produced in events that occur considerably less frequently than do Type II SNe ${ }^{17}$ (SNII). This sounds a little like collapsars. Nonetheless, any arguments in favor of collapsars as the source of 2 nd peak elements are speculative at best. A clear and convincing observational test is needed.

One possible test is a correlation between abundances of second peak $\mathrm{r}$ elements and nucleosynthetically unrelated elements ejected in the collapsar explosion. To illustrate this idea, suppose that we somehow knew that collapsars make a lot of, say, ${ }^{45} \mathrm{Sc}$. A convincing correlation between $[\mathrm{Sc} / \mathrm{Fe}]$ and $[\mathrm{Ag} / \mathrm{Fe}]$ in metal-poor stars would be evidence that collapsars make silver. Of course, this idea can only work if other astrophysical events (like SNII) can't explain the correlation. To address this, we need a detailed understanding of what it is that collapsars synthesize.

It turns out that even though there are uncertainties concerning the detailed inner-workings of collapsars, a lot can be said about nucleosynthesis in these events by making two plausible assumptions ${ }^{18}$. These are i) that observed nickel is synthesized in a wind from the accretion disk and ii) that the wind provides most of the energy for exploding the star. With these assumptions the only really important unknown is whether $Y_{e}$ in the wind is very close to $1 / 2$, or if instead $Y_{e} \gtrsim 0.51$. The assumption that observed ${ }^{56} \mathrm{Ni}$ comes from the wind means that $Y_{e} \gtrsim 0.485$, since more neutron-rich outflows synthesize very little ${ }^{56} \mathrm{Ni}$.

Figure 3 shows results of nucleosynthesis calculations for collapsar winds characterized by $Y_{e}=0.51$ and different dynamic time scales and entropies. In each panel $s$ is the entropy per baryon and $X(\mathrm{Ni})$ is the mass fraction of ${ }^{56} \mathrm{Ni}$ in the wind. A range of $s \sim 20-50$ is thought to approximately bracket the plausible range of entropies found in these winds. The two different calculations shown for each entropy are for the estimated range of plausible dynamic time scales possible in these winds. Though there are some differences between the panels, it is clear that some isotopes - including the only stable Sc isotope - are abundantly synthesized in every case. ${ }^{46,49} \mathrm{Ti}$ are also abundant, but they make only modest contributions to the total Ti inventory, and it is hard to get isotopic ratios observationally. Cal- 
culations for winds with $Y_{e}$ very close to $1 / 2$ show that the most abundant elements are ${ }^{63} \mathrm{Cu}$ and ${ }^{64} \mathrm{Zn}$. We'll concentrate on the case $Y_{e} \gtrsim 0.505$. For $Y_{e}$ very close to $1 / 2$ one can just substitute ${ }^{64} \mathrm{Zn}$ for ${ }^{45} \mathrm{Sc}$ in what follows.
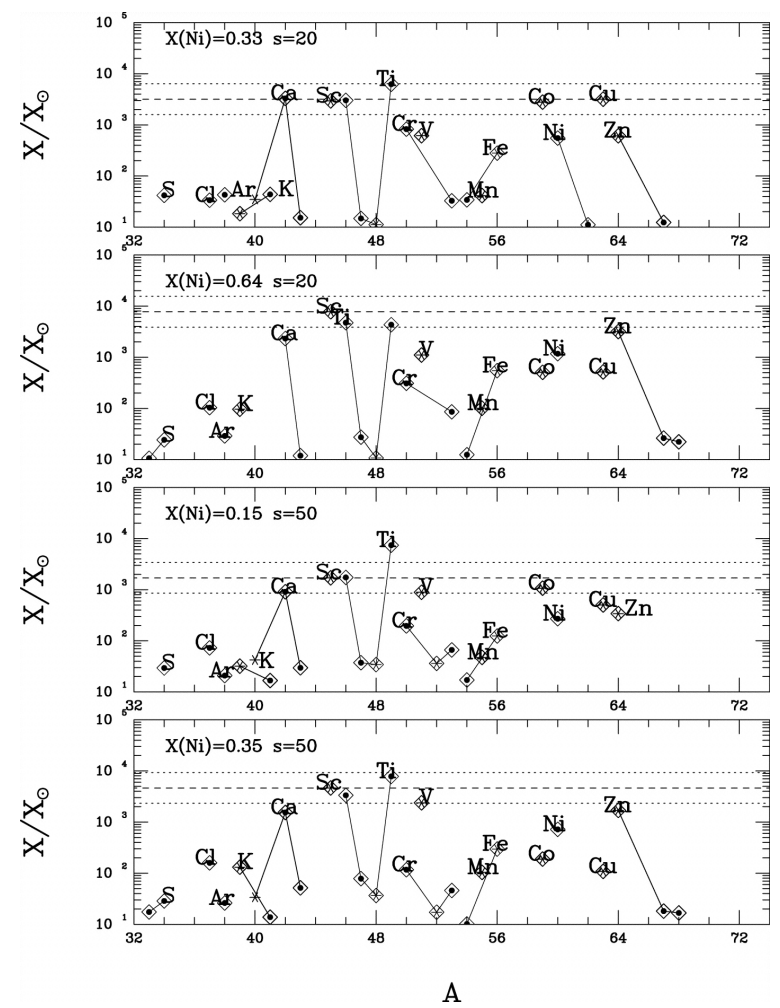

Figure 3. Nucleosynthesis in different collapsar winds. For all panels shown here $Y_{e}=$ 0.51 . The range of entropies and dynamic time scales represented by the different panels is thought to approximately bracket the range of conditions for collapsar winds that efficiently synthesize ${ }^{56} \mathrm{Ni}$.

If a wind is responsible for the $\sim 1 / 2 M_{\odot}$ of $\mathrm{Ni}$ observed in association with GRBs, then the total wind mass is $M_{\text {wind }} \approx 1 M_{\odot}$ if $X(\mathrm{Ni}) \sim 0.5$. The production factor of Sc is

$$
O\left({ }^{45} \mathrm{Sc}\right) \equiv \frac{M_{\mathrm{wind}}}{M_{\mathrm{ej}}} \frac{X(\mathrm{Sc})}{X_{\odot}(\mathrm{Sc})} \approx \frac{1 M_{\odot}}{20 M_{\odot}} \frac{X(\mathrm{Sc})}{X_{\odot}(\mathrm{Sc})} \sim 300 .
$$

Here $M_{\mathrm{ej}}$ is the total mass ejected in the collapsar explosion. For reference, nuclei that owe their origin to Type II SNe have production factors $O \sim$ 
$10-20$. This means that collapsars make $10-50$ times more Sc or Zn than typical $\mathrm{SNe}$ can. If collapsars are responsible for $\mathrm{Ag}$ synthesis, then $O(\mathrm{Ag})$ must also be $\sim 300-1000$, depending on the collapsar rate. In this case, there should be observable correlations between $\mathrm{Ag}$ and $\mathrm{Sc}$ (or $\mathrm{Zn}$ ) in metal-poor stars.

There are only 4 stars I'm aware of for which both $\mathrm{Ag}$ and $\mathrm{Sc}$ (or Zn) abundances are known (HD2665, HD6755, HD103095, CS22892-052). For the $\mathrm{HD}$ stars there is a modest correlation between $[\mathrm{Ag} / \mathrm{Fe}]$ and $[\mathrm{Sc} / \mathrm{Fe}]$ (or $[\mathrm{Zn} / \mathrm{Fe}])$. For CS22892-052 [Ag/Fe] 1, while [Sc/Fe] and $[\mathrm{Zn} / \mathrm{Fe}]$ are close to zero. It will be interesting to see if future observations can confirm or rule out the idea of collapsars as the source of 2 nd peak r-process elements.

\section{Acknowledgements}

This research has been supported through a grant from the US DOE Program for Scientific Discovery through Advanced Computing (SciDAC; DEFC-01ER41176). This work was performed under the auspices of the US DOE by the University of California, Lawrence Livermore National Laboratory under contract W-7405-ENG-48.

\section{References}

1. Woosley, S.E. \& Heger, A. 2003, ApJ, submitted (astro-ph/0309165)

2. Maeda, et al. 2003, ApJ, 593, 931

3. Price, P. A. et al. 2003, ApJ, 589, 838

4. Zeh, A., Klose, S. \& Hartmann, D.H. 2004, ApJ, accepted

5. Woosley, S.E. 1993, ApJ, 405, 273

6. MacFadyen, A.I. \& Woosley, S.E. 1999, ApJ, 524, 262

7. Maeda, K. \& Nomoto, K. 2003, ApJ, 598, 1163

8. Pruet, J., Thompson, T.A. \& Hoffman, R.D. 2004, ApJ, in press

9. Pruet, J., Woosley, S.E. \& Hoffman, R.D. 2003, ApJ, 586, 1254

10. Popham, R., Woosley, S.E. \& Fryer, C.L. 1999, ApJ, 518, 356

11. Beloborodov, A.M. 2003, ApJ, 588, 931

12. Surman, R. \& McLaughlin, G.C. 2004, ApJ, 603, 611

13. Mazzali, P., et. al. 2003, ApJL, 599, 95

14. Qian, Y.-Z. \& Woosley, S.E. 1996, ApJ, 471, 331

15. Balbus, S. A. \& Hawley, J. F. 1998, Rev. Mod. Phys., 70, No. 1

16. Heger, A., Fryer, C.L., Woosley, S.E., Langer, N. \& Hartmann, D.H. 2003, ApJ, 591, 288

17. Qian, Y.-Z. \& Wasserburg, G. J. 2000, Phys. Reps., 333, 77

18. Pruet, J., Surman, R. \& McLaughlin, G.C. 2004, ApJL, 602, 101 